\title{
Mazur-Ulam type theorems for fuzzy normed spaces
}

\author{
J. J. Font, J. Galindo, S. Macario*, M. Sanchis
}

Departament de Matemàtiques and Institut Universitari de Matemàtiques i Aplicacions de Castelló (IMAC), Universitat Jaume I, Avda. Sos Baynat, s/n, 12071 Castelló, Spain.

\begin{abstract}
In this paper, we provide Mazur-Ulam type results for (not necessarily surjective) maps preserving equality of fuzzy distance defined between two fuzzy normed spaces. Our main goal is to study the additivity of such generalizations of fuzzy isometries. As in the classical case, the fuzzy strict convexity of the target space will play an important role. (c)2017 All rights reserved.
\end{abstract}

Keywords: Mazur-Ulam theorem, fuzzy normed spaces, strict convexity.

2010 MSC: 46B04, 46S40.

\section{Introduction}

The classical Mazur-Ulam theorem states that surjective isometries defined between normed spaces are affine maps. It was proved by Mazur and Ulam in 1932 ([11]) in response to an issue raised by Stefan Banach. When surjectivity is replaced by other conditions, we get a Mazur-Ulam type theorem. For example, Baker ([3]) proved that the result remains true if we consider a (not necessarily onto) isometry between a real normed space and a strictly convex real normed space. Another direction was provided by Vogt ([15]), who replaced isometries by the more general notion of equality of distance preserving maps (see also [12]). Our aim in this paper is to achieve some results of this kind for fuzzy normed spaces.

In [6], following the ideas of Vogt ([15]), who in turn was inspired by von Neumann and Schoenberg ([16]), the authors introduced a generalization of the concept of fuzzy isometry and obtained a MazurUlam type theorem in a fuzzy context by proving the additivity of surjective maps preserving equality of fuzzy distance.

In this paper we provide Mazur-Ulam type results for such generalized fuzzy isometries defined between two fuzzy normed spaces without assuming surjectivity. Our main goal is, therefore, to study the additivity of (not necessarily onto) maps preserving equality of fuzzy distance. We also derive an alternative result to the one provided by Rui ([12]) in the classical setting from our results in a fuzzy context.

We first review some basic notions concerning fuzzy metrics and fuzzy norms.

\footnotetext{
${ }^{*}$ Corresponding author

Email addresses: font@uji.es (J. J. Font), jgalindo@uji.es (J. Galindo), macario@uji.es (S. Macario), sanchis@uji.es (M. Sanchis)

doi:10.22436/jnsa.010.08.41
}

Received 2017-07-19 


\subsection{Fuzzy metrics and fuzzy norms}

Kramosil and Michalek in 1975 introduced the notion of fuzzy metric space by generalizing the concept of probabilistic metric space to the fuzzy situation. The idea of introducing a fuzzy norm on a vector space was first used by Katzaras ([9]) in 1984. Eight years later, Felbin ([5]) offered an alternative definition which induced a fuzzy metric of Kaleva-Seikkala type ([8]). Cheng and Mordeson ([4]) shortly afterwards defined another type of fuzzy norm with associated fuzzy metric of Kramosil-Michalek type.

We shall begin with the definition of fuzzy metric space in the sense of Kramosil and Michalek ([10]). Following [14], a continuous t-norm is a binary operation $*:[0,1] \times[0,1] \rightarrow[0,1]$ which satisfies the following conditions: (i) $*$ is associative and commutative, (ii) $*$ is continuous, (iii) $a * 1=a$ for every $a \in[0,1]$, and (iv) $a * b \leqslant c * d$ whenever $a \leqslant c$ and $b \leqslant d$, with $a, b, c, d \in[0,1]$.

The three most used continuous t-norms are the usual product $a * b=a \cdot b$, the Lukasiewicz t-norm $a *_{L} b=\max \{a+b-1,0\}$, and the minimum $t$-norm $a \wedge b=\min \{a, b\}$. It is worth noting that $* \leqslant \wedge$ for every continuous t-norm.

Definition 1.1 ([10]). A fuzzy metric (in the sense of Kramosil and Michalek) on a set $X$ is a pair $(M, *)$ such that $M$ is a fuzzy set in $X \times X \times[0, \infty)$ and $*$ is a continuous t-norm satisfying for all $x, y, z \in X$ and $t, s>0$ :

(i) $M(x, y, 0)=0$;

(ii) $M(x, y, t)=1$ for all $t>0$ if and only if $x=y$;

(iii) $M(x, y, t)=M(y, x, t)$;

(iv) $M(x, y, t) * M(y, z, s) \leqslant M(x, z, t+s)$; and

(v) $M(x, y, \cdot):[0,+\infty) \rightarrow[0,1]$ is a left continuous function.

By a fuzzy metric space (in the sense of Kramosil and Michalek) we mean a triple $(X, M, *)$ such that $X$ is a set and $(M, *)$ is a fuzzy metric on $X$. Recall that every fuzzy metric $(M, *)$ on $X$ induces a topology $\tau_{M}$ on $X$, which has as a base the family of open sets of the form $\left\{B_{M}(x, \varepsilon, t): x \in X, \varepsilon \in(0,1), t>0\right\}$, where $B_{M}(x, \varepsilon, t)=\{y \in X: M(x, y, t)>1-\varepsilon\}$ for all $x \in X, \varepsilon \in(0,1)$ and $t>0$. These sets will be called open balls for $M$.

We will work with the following definition of a fuzzy norm, which is just the one given by ChengMordeson, taking as t-norm $*=\wedge$.

Definition 1.2. Let $X$ be a real linear space. A function $N: X \times[0,+\infty) \longrightarrow[0,1]$ is said to be a fuzzy norm on $X$ if, for all $x, y \in X$ and all $t, s>0$, it satisfies the following:

(N1) $\mathrm{N}(\mathrm{x}, 0)=0$;

(N2) $x=0$ if, and only if, $N(x, t)=1$ for all $t>0$;

(N3) $N(k x, t)=N(x, t /|k|)$ for all $k \in \mathbb{R}, k \neq 0$;

(N4) $N(x+y, t+s) \geqslant \min \{N(x, t), N(y, s)\}$;

(N5) $N(x,-):(0,+\infty) \rightarrow[0,1]$ is a left continuous function and $\lim _{t \rightarrow \infty} N(x, t)=1$.

The pair $(X, N)$ will be called a fuzzy normed space.

To gain flexibility, some additional conditions are often imposed in the above definition. A fuzzy normed space, for instance, is said to satisfy condition (N6), see [2], if $N(x, t)>0$ for all $t>0$ yields $x=0$. Also a fuzzy normed space is said to satisfy condition (N7) if for $x \neq 0, N(x, \cdot)$ is a continuous function on $(0,+\infty)$ which is strictly increasing on $\{t \in(0,+\infty): 0<N(x, t)<1\}$.

Every fuzzy norm $(N, \wedge)$ over a real linear space $X$, induces a fuzzy metric as follows:

$$
M_{N}(x, y, t)=N(x-y, t), \text { for all } x, y \in X, t \geqslant 0,
$$

and, then, for every $x \in X$, the countable collection of balls $\left\{B_{M_{N}}(x, 1 / n, 1 / n): n=2,3, \ldots\right\}$ forms a fundamental system of neighborhoods of $x$ for the topology induced by $M_{N}$ (see [7]) making $(X, N)$ into a Hausdorff metrizable topological vector space. 
It is well-known that classical (real) normed spaces are strictly included in the class of fuzzy normed spaces (see [1] for an example of a fuzzy normed space which is not a normable space) and that (N2) and (N4) imply that, for a fixed $x \in X$, the function $N(x, \cdot)$ is nondecreasing.

Example 1.3. Let $(X,\|\cdot\|)$ be a real normed space.

(a) Let $\mathrm{N}_{\mathrm{s}}: X \times\left[0,+\infty\left[\rightarrow[0,1]\right.\right.$ be given by $\mathrm{N}_{\mathrm{s}}(x, 0)=0$ for all $x \in X$ and

$$
\mathrm{N}_{\mathrm{s}}(x, \mathrm{t})=\frac{\mathrm{t}}{\mathrm{t}+\|x\|}
$$

for all $x \in X$ and $t>0$. Then, $N_{s}$ is a fuzzy norm on $X$ which is called the standard fuzzy norm induced by $\|\cdot\|$.

(b) Let $\mathrm{N}_{01}: \mathrm{X} \times[0,+\infty[\rightarrow[0,1]$ given by

$$
N_{01}(x, t)= \begin{cases}0, & \text { if } t \leqslant\|x\|, \\ 1, & \text { if } t>\|x\| .\end{cases}
$$

Then, $\mathrm{N}_{01}$ is a fuzzy norm on $\mathrm{X}$. This fuzzy norm will be called the discrete fuzzy norm induced by $\|\cdot\|$.

In both cases, the topology defined by the fuzzy norm coincides with the topology of the original norm on $X$.

We point out that the standard fuzzy norm satisfies the condition (N7) but not (N6) and the discrete fuzzy norm satisfies (N6) but not (N7).

\section{Preliminaries}

Definition 2.1. Let $(X, N)$ be a fuzzy normed space. We say that $(X, N)$ is strictly convex if whenever

$$
N\left(\frac{x+y}{2}, t\right)=N(x, t)=N(y, t) \text { for some } t>0, \text { then } x=y .
$$

Let us recall here that a classical real normed space $(X,\|\cdot\|)$ is strictly convex if given $a, b \in X$ such that $\left\|\frac{\mathrm{a}+\mathrm{b}}{2}\right\|=\|\mathrm{a}\|=\|\mathrm{b}\|$, then $\mathrm{a}=\mathrm{b}$.

It can be easily checked that if $(X,\|\cdot\|)$ is a strictly convex normed space, then $\left(X, N_{s}\right)$ is also a strictly convex fuzzy normed space. However, this fact is no longer true for other fuzzy norms. For instance, let us consider the fuzzy normed space $\left(\mathbb{R}, N_{01}\right)$ induced by the strictly convex normed space $(\mathbb{R},|\cdot|)$. For $x=1, y=-1$, and $t=2$, we get then that $N_{01}(x, t)=N_{01}(y, t)=N_{01}\left(\frac{x+y}{2}, t\right)=1$, while $x \neq y$. That means that the property of being fuzzy strictly convex is a 'geometrical' property rather than a topological one.

Definition 2.2. Let $(X, N)$ and $\left(Y, N^{\prime}\right)$ be two fuzzy normed spaces.

1. We say that $f: X \longrightarrow Y$ preserves equality of fuzzy distance if given $x, y, \tilde{x}, \tilde{y} \in X$ and $t>0$ with $N(x-y, t)=N(\tilde{x}-\tilde{y}, t)$, then $N^{\prime}(f(x)-f(y), t)=N^{\prime}(f(\tilde{x})-f(\tilde{y}), t)$.

2. It is said that $f: X \longrightarrow Y$ is a fuzzy isometry if $N^{\prime}(f(x)-f(y), t)=N(x-y, t)$ for all $x, y \in X$ and $t>0$.

3. It is said that $f: X \longrightarrow Y$ is segment-preserving if, given $x, y \in X, f([x, y]) \subseteq[f(x), f(y)]$ where $[x, y]$ stands for the segment $\{\lambda x+(1-\lambda) y: 0 \leqslant \lambda \leqslant 1\}$.

Remark 2.3. It can be checked that if $f:(X, N) \longrightarrow\left(Y, N^{\prime}\right)$ preserves equality of fuzzy distance, then it is associated, for each $t>0$, to a function $\rho_{t}: D_{t} \subseteq[0,1] \longrightarrow[0,1]$ such that

$$
N^{\prime}(f(x)-f(y), t)=\rho_{t}(N(x-y, t)) \text {. }
$$


It is then apparent that every fuzzy isometry preserves equality of fuzzy distance by taking $\rho=\mathrm{Id}$. However both concepts are different as the following example shows.

Let $(X,\|\cdot\|)$ be a real normed space and define $f:\left(X, N_{s}\right) \rightarrow\left(X, N_{s}\right)$ by $f(x)=2 x$. This map is not a fuzzy isometry but preserves equality of fuzzy distance in an even more general way. Indeed, if we assume $N_{s}(x-y, t)=N_{s}\left(\tilde{x}-\tilde{y}, t^{\prime}\right)$ for some $x, y, \tilde{x}, \tilde{y} \in X$ and $t, t^{\prime}>0$, then we infer $\frac{\|x-y\|}{t}=\frac{\|\tilde{x}-\tilde{y}\|}{t^{\prime}}$. Consequently,

$$
N_{s}(f(x)-f(y), t)=N_{s}(2 x-2 y, t)=\frac{t}{t+2\|x-y\|}=\frac{1}{1+2 \frac{\|x-y\|}{t}}=\frac{1}{1+2 \frac{\|\tilde{x}-\tilde{y}\|}{t^{\prime}}}=N_{s}\left(f(\tilde{x})-f(\tilde{y}), t^{\prime}\right) .
$$

In connection with this example, it is worth noting that functions preserving equality of fuzzy distance enjoy this extended property when they are either positively homogeneous or affine (in particular, linear). To be precise, suppose that $f$ is a positively homogeneous function from a fuzzy normed space $(X, N)$ into a fuzzy normed space $\left(Y, N^{\prime}\right)$ which preserves equality of fuzzy distance. We show that $N^{\prime}(f(x)-$ $f(y), t)=N^{\prime}\left(f(\tilde{x})-f(\tilde{y}), t^{\prime}\right)$ whenever $N(x-y, t)=N\left(\tilde{x}-\tilde{y}, t^{\prime}\right)\left(t, t^{\prime}>0\right)$. In fact, let $k>0$ be such that $t=k t^{\prime}$. Then $N(x-y, t)=N\left(k \tilde{x}-k \tilde{y}, k t^{\prime}\right)$ so that $N^{\prime}(f(x)-f(y), t)=N^{\prime}\left(f(k \tilde{x})-f(k \tilde{y}), k t^{\prime}\right)$. Being $f$ positively homogeneous, we have

$$
N^{\prime}(f(x)-f(y), t)=N^{\prime}\left(k(f(\tilde{x})-f(\tilde{y})), k t^{\prime}\right)=N^{\prime}\left(f(\tilde{x})-f(\tilde{y}), t^{\prime}\right) .
$$

The case of $f$ being affine runs along a similar pattern.

\section{The results}

Lemma 3.1. Let $(\mathrm{X}, \mathrm{N})$ be a fuzzy normed space. Let $\mathrm{x}, \mathrm{y} \in \mathrm{X}$ and $\mathrm{t}>0$.

1. If $X$ is strictly convex, then $z=\frac{x+y}{2}$ is the unique element of $X$ satisfying

$$
\mathrm{N}(x-z, \mathrm{t})=\mathrm{N}(z-\mathrm{y}, \mathrm{t})=\mathrm{N}(x-y, 2 \mathrm{t}) .
$$

2. If $(X, N)$ satisfies (N7), then $z=\frac{x+y}{2}$ is the unique element in the segment $[x, y]$ satisfying

$$
\mathrm{N}(x-z, \mathrm{t})=\mathrm{N}(z-y, t)=N(x-y, 2 t) .
$$

Proof.

(1) Firstly,

$$
N\left(x-\frac{x+y}{2}, t\right)=N\left(\frac{x-y}{2}, t\right)=N(x-y, 2 t)
$$

and

$$
N\left(\frac{x+y}{2}-y, t\right)=N\left(\frac{x-y}{2}, t\right)=N(x-y, 2 t) .
$$

Let us suppose there is another $z^{\prime} \in X$ such that

$$
N\left(x-z^{\prime}, t\right)=N\left(z^{\prime}-y, t\right)=N(x-y, 2 t) .
$$

Indeed,

$$
\mathrm{N}\left(x-\frac{z+z^{\prime}}{2}, \mathrm{t}\right)=\mathrm{N}\left(2 x-z-z^{\prime}, 2 \mathrm{t}\right) \geqslant \min \left\{\mathrm{N}(x-z, \mathrm{t}), \mathrm{N}\left(x-z^{\prime}, \mathrm{t}\right)\right\}=\mathrm{N}(x-y, 2 \mathrm{t})
$$

and

$$
N\left(\frac{z+z^{\prime}}{2}-y, t\right)=N\left(z+z^{\prime}-2 y, 2 t\right) \geqslant \min \left\{N(z-y, t), N\left(z^{\prime}-y, t\right)\right\}=N(x-y, 2 t)
$$


If (3.1) and (3.2) were both strict, then

$$
\begin{aligned}
\mathrm{N}(x-y, 2 t)=N\left(x-\frac{z+z^{\prime}}{2}+\frac{z+z^{\prime}}{2}-y, 2 t\right) & \geqslant \min \left\{N\left(x-\frac{z+z^{\prime}}{2}, t\right), N\left(\frac{z+z^{\prime}}{2}-y, t\right)\right\} \\
& >N(x-y, 2 t),
\end{aligned}
$$

which is a contradiction. Hence, we can assume, with no loss of generality, that

$$
\mathrm{N}\left((x-z)+\left(x-z^{\prime}\right), 2 \mathrm{t}\right)=\min \left\{\mathrm{N}(x-z, \mathrm{t}), \mathrm{N}\left(x-z^{\prime}, \mathrm{t}\right)\right\} .
$$

Besides, since $N(x-z, t)=N\left(x-z^{\prime}, t\right)$, we get

$$
\mathrm{N}\left(\frac{(x-z)+\left(x-z^{\prime}\right)}{2}, \mathrm{t}\right)=\mathrm{N}(x-z, \mathrm{t})=\mathrm{N}\left(x-z^{\prime}, \mathrm{t}\right)
$$

and we infer by strictly convexity that $x-z=x-z^{\prime}$ and so $z=z^{\prime}$.

(2) Let us suppose that there is $z^{\prime} \in[x, y]$, say $z^{\prime}=\lambda x+(1-\lambda) y$ for some $\lambda \in[0,1]$, such that

$$
N(x-(\lambda x+(1-\lambda) y), t)=N(\lambda x+(1-\lambda) y-y, t)=N(x-y, 2 t) .
$$

Hence

$$
N\left(x-y, \frac{t}{1-\lambda}\right)=N\left(x-y, \frac{t}{\lambda}\right)=N(x-y, 2 t)
$$

and, by (N7), we infer

$$
\frac{1}{1-\lambda}=\frac{1}{\lambda}=2
$$

which yields $\lambda=\frac{1}{2}$ as was to be proved.

Theorem 3.2. Let $(\mathrm{X}, \mathrm{N})$ and $\left(\mathrm{Y}, \mathrm{N}^{\prime}\right)$ be fuzzy normed spaces and let $\mathrm{f}: \mathrm{X} \longrightarrow \mathrm{Y}$ preserve equality of fuzzy distance and satisfy the equalities $\mathrm{f}(0)=0$ and

$$
N^{\prime}\left(f\left(\frac{x}{2}\right), t\right)=N^{\prime}\left(\frac{f(x)}{2}, t\right)
$$

for all $\mathrm{x} \in \mathrm{X}$ and $\mathrm{t}>0$.

1. If $\left(\mathrm{Y}, \mathrm{N}^{\prime}\right)$ is strictly convex, then $\mathrm{f}$ is additive.

2. If $\left(\mathrm{Y}, \mathrm{N}^{\prime}\right)$ satisfies (N7) and $\mathrm{f}$ is segment-preserving, then $\mathrm{f}$ is additive.

Proof.

(1) Assume first that $\left(Y, N^{\prime}\right)$ is strictly convex. For all $x \in X$ and $t>0$

$$
\rho_{t}(N(x, 2 t))=N^{\prime}\left(f\left(\frac{x}{2}\right), t\right)=N^{\prime}\left(\frac{f(x)}{2}, t\right)=\rho_{2 t}(N(x, 2 t)) .
$$

Hence, for all $x, y \in X$ and $t>0$, we have

$$
\begin{aligned}
N^{\prime}\left(f\left(\frac{x+y}{2}\right)-f(y), t\right) & =\rho_{t}\left(N\left(\frac{x+y}{2}-y, t\right)\right) \\
& =\rho_{t}\left(N\left(\frac{x-y}{2}, t\right)\right) \\
& =\rho_{t}(N(x-y, 2 t)) \\
& =\rho_{2 t}(N(x-y, 2 t)) \\
& =N^{\prime}(f(x)-f(y), 2 t)
\end{aligned}
$$


and, similarly,

$$
\begin{aligned}
N^{\prime}\left(f(x)-f\left(\frac{x+y}{2}\right), t\right) & =\rho_{t}\left(N\left(x-\frac{x+y}{2}, t\right)\right) \\
& =\rho_{t}\left(N\left(\frac{x-y}{2}, t\right)\right) \\
& =\rho_{t}(N(x-y, 2 t)) \\
& =\rho_{2 t}(N(x-y, 2 t)) \\
& =N^{\prime}(f(x)-f(y), 2 t) .
\end{aligned}
$$

By Lemma $3.1(1)$, we infer that $f\left(\frac{x+y}{2}\right)=\frac{f(x)+f(y)}{2}$ for every $x, y \in X$. Since $f(0)=0$, we have $f(x)=$ $f\left(\frac{2 x+0}{2}\right)=\frac{f(2 x)}{2}$. Hence, $f(x+y)=f\left(\frac{2 x+2 y}{2}\right)=\frac{f(2 x)}{2}+\frac{f(2 y)}{2}=f(x)+f(y)$.

(2) Assume next that $\left(\mathrm{Y}, \mathrm{N}^{\prime}\right)$ satisfies (N7) and that $f$ is segment-preserving. As in (1), we have

$$
N^{\prime}\left(f\left(\frac{x+y}{2}\right)-f(y), t\right)=N^{\prime}\left(f(x)-f\left(\frac{x+y}{2}\right), t\right)=N^{\prime}(f(x)-f(y), 2 t) .
$$

Hence, by Lemma $3.1(2)$, we infer that $f\left(\frac{x+y}{2}\right)=\frac{f(x)+f(y)}{2}$ for every $x, y \in X$ and the rest of the proof follows as in (1).

Remark 3.3. The assumptions of strict convexity and segment preservation in the above theorem are not redundant as the following example shows.

Let us consider $\mathbb{R}$ endowed with the standard fuzzy norm $\mathrm{N}_{\mathrm{s}}$ induced by $|\cdot|$ and $\mathbb{R}^{2}$ with the standard fuzzy norm $N_{s}^{\prime}$ induced by the norm $\|(x, y)\|=\max \{|x|,|y|\}$. It suffices to remark that $N_{s}^{\prime}((1,1), t)=$ $N_{s}^{\prime}((-1,1), t)=N_{s}^{\prime}((0,1), t)$ to check that the fuzzy normed space $\left(\mathbb{R}^{2}, N_{s}^{\prime}\right)$ is not strictly convex. Besides, it satisfies (N7). It is a routine matter to verify that the map $f:\left(\mathbb{R}, N_{s}\right) \longrightarrow\left(\mathbb{R}^{2}, N_{s}^{\prime}\right)$ defined as $f(x)=$ $(x,|x|)$ is not segment-preserving but preserves equality of fuzzy distance, $f(0)=0$ and $N_{s}^{\prime}\left(f\left(\frac{x}{2}\right), t\right)=$ $N_{s}^{\prime}\left(\frac{f(x)}{2}, t\right)$ for all $x \in \mathbb{R}$ and $t>0$. However $f$ is not additive.

Corollary 3.4. Let $(\mathrm{X}, \mathrm{N})$ and $\left(\mathrm{Y}, \mathrm{N}^{\prime}\right)$ be fuzzy normed spaces and let $\mathrm{f}: \mathrm{X} \longrightarrow \mathrm{Y}$ be a fuzzy isometry. Then $\mathrm{f}(\mathrm{X})-\mathrm{f}(0)$ is additive if either $\left(\mathrm{Y}, \mathrm{N}^{\prime}\right)$ is strictly convex or $\left(\mathrm{Y}, \mathrm{N}^{\prime}\right)$ satisfies (N7) and $\mathrm{f}$ is segment-preserving.

Proof. Let $g(x)=f(x)-f(0)$, which is a fuzzy isometry. Furthermore, for all $x \in X$ and $t>0$,

$$
N^{\prime}\left(g\left(\frac{x}{2}\right), t\right)=N(x, 2 t)=N^{\prime}\left(\frac{g(x)}{2}, t\right)
$$

and the result follows from Theorem 3.2.

Remark 3.5. In [13] and [16], Schoenberg and von Neumann generalized the concept of isometry by introducing and studying the following maps.

A map $f:\left(X,\|\cdot\|_{X}\right) \longrightarrow\left(Y,\|\cdot\|_{Y}\right)$ preserves equality of distance if

$$
\|x-y\|_{X}=\|\tilde{x}-\tilde{y}\|_{X} \Rightarrow\|f(x)-f(y)\|_{Y}=\|f(\tilde{x})-f(\tilde{y})\|_{Y}
$$

or, equivalently, there exists $\rho:\left[0,+\infty\left[\longrightarrow\left[0,+\infty\left[\right.\right.\right.\right.$ such that $\|f(x)-f(y)\|_{Y}=\rho\left(\|x-y\|_{X}\right)$. In [15], Vogt provided a Mazur-Ulam type result for surjective maps preserving equality of distance, which was recently extended to the context of fuzzy normed spaces in [6]. Inspired by Baker's generalization of Mazur-Ulam theorem to a non-surjective setting ([3]), Rui ([12]) proved the following: if $f: X \longrightarrow Y, Y$ being strictly convex, preserves equality of distance, satisfies $f(0)=0$, and has a segment contained in its range, then $f$ is additive.

In the next corollary, we provide an alternative result based on Theorem 3.2, that is, we obtain a result for classical normed spaces from the fuzzy setting. 
Corollary 3.6. Assume $\mathrm{f}:\left(\mathrm{X},\|\cdot\|_{\mathrm{X}}\right) \longrightarrow\left(\mathrm{Y},\|\cdot\|_{\mathrm{Y}}\right)$ preserves equality of distance, $\mathrm{f}(0)=0$, and

$$
\left\|f\left(\frac{x}{2}\right)\right\|_{Y}=\left\|\frac{f(x)}{2}\right\|_{Y} .
$$

If either $\left(\mathrm{Y},\|\cdot\|_{\mathrm{Y}}\right)$ is strictly convex or $\mathrm{f}$ is segment-preserving, then $\mathrm{f}$ is additive.

Proof. Let $\mathrm{N}_{s}$ (resp. $\mathrm{N}_{s}^{\prime}$ ) be the standard fuzzy norm induced by $\|\cdot\|_{X}$ (resp. $\|\cdot\|_{Y}$ ). It is apparent that the map $f:\left(X, N_{s}\right) \longrightarrow\left(Y, N_{s}^{\prime}\right)$ satisfies

$$
N_{s}(x-y, t)=N_{s}(\tilde{x}-\tilde{y}, t) \Rightarrow N_{s}^{\prime}(f(x)-f(y), t)=N_{s}^{\prime}(f(\tilde{x})-f(\tilde{y}), t)
$$

for all $x, y \in X$ and $t>0$, which is to say that $f$ preserves equality of fuzzy distance. Furthermore

$$
N_{s}^{\prime}\left(f\left(\frac{x}{2}\right), t\right)=N_{s}^{\prime}\left(\frac{f(x)}{2}, t\right)
$$

for all $x \in X$ and $t>0$.

If we assume the strict convexity of $\left(Y,\|\cdot\|_{Y}\right)$, then $\left(Y, N_{s}^{\prime}\right)$ is also fuzzy strictly convex (see Section 2) and the result follows from Theorem $3.2(1)$.

On the other hand, if we assume that $\mathrm{f}$ is segment-preserving, the result follows from Theorem 3.2 (2) since $\left(\mathrm{Y}, \mathrm{N}_{\mathrm{s}}^{\prime}\right)$ satisfies $(\mathrm{N} 7)$.

Definition 3.7. Let $(X, N)$ and $\left(Y, N^{\prime}\right)$ be fuzzy normed spaces. We say that a map $f: X \longrightarrow Y$ is normadditive if, given any finite subset $\left\{x_{1}, \ldots, x_{n}\right\}$ of $X$,

$$
N^{\prime}\left(f\left(\sum_{i=1}^{n} x_{i}\right), t\right)=N^{\prime}\left(\sum_{i=1}^{n} f\left(x_{i}\right), t\right)
$$

for all $t>0$.

Theorem 3.8. Let $(\mathrm{X}, \mathrm{N})$ and $\left(\mathrm{Y}, \mathrm{N}^{\prime}\right)$ be fuzzy normed spaces. Assume also that $\left(\mathrm{Y}, \mathrm{N}^{\prime}\right)$ is strictly convex and let $\mathrm{f}: \mathrm{X} \longrightarrow \mathrm{Y}$ be a norm additive map with $\mathrm{f}(0)=0$. Then $\mathrm{f}$ is additive.

Proof. Since, for all $x \in X$,

$$
1=N^{\prime}(0, t)=N^{\prime}(f(x-x), t)=N^{\prime}(f(x)+f(-x), t) \text { for all } t>0,
$$

we have $f(-x)=-f(x)$ for all $x \in X$. On the other hand,

$$
\begin{aligned}
N^{\prime}\left(f(x+y)-\frac{1}{2} f(x), t\right) & =N^{\prime}(2 f(x+y)-f(x), 2 t) \\
& =N^{\prime}(f(x+y+y), 2 t) \\
& =N^{\prime}(f(x+y)+f(y), 2 t) \\
& =N^{\prime}\left(\frac{f(x+y)+f(y)}{2}, t\right) .
\end{aligned}
$$

Furthermore,

$$
\begin{aligned}
N^{\prime}\left(\frac{1}{2} f(x)+f(y), t\right) & =N^{\prime}(f(x)+2 f(y), 2 t) \\
& =N^{\prime}(f(x+y+y), 2 t) \\
& =N^{\prime}(f(x+y)+f(y), 2 t) \\
& =N^{\prime}\left(\frac{f(x+y)+f(y)}{2}, t\right)
\end{aligned}
$$


Taking $u:=f(x+y)-\frac{1}{2} f(x)$ and $v:=\frac{1}{2} f(x)+f(y)$, from above, it is clear that

$$
\mathrm{N}^{\prime}(\mathrm{u}, \mathrm{t})=\mathrm{N}^{\prime}(v, \mathrm{t})=\mathrm{N}^{\prime}\left(\frac{\mathrm{u}+v}{2}, \mathrm{t}\right)
$$

and strictly convexity applies to get $u=v$, which means $f(x+y)=f(x)+f(y)$.

Question 3.9. In [3], Baker characterized strictly convex spaces $Y$ among real normed spaces as those for which any isometry $f: X \rightarrow Y$ from a normed space $X$ into $Y$ with $f(0)=0$ is additive.

A similar question can be raised in the fuzzy setting. Since necessity follows from Theorem 3.2, the question here is: given a non-strictly convex fuzzy normed space $Y$, can we find a fuzzy normed space $X$ and a non-additive map preserving equality of fuzzy distance $f: X \longrightarrow Y$, with $f(0)=0$ ?

\section{Acknowledgment}

This research is supported by Spanish Government (MTM2016-77143-P) and Universitat Jaume I (P11B2014-35).

\section{References}

[1] C. Alegre, S. Romaguera, Characterizations of metrizable topological vector spaces and their asymmetric generalizations in terms of fuzzy (quasi-) norms, Fuzzy Sets and Systems, 161 (2010), 2181-2192. 1.1

[2] T. Bag, S. K. Samanta, Finite dimensional fuzzy normed linear spaces, J. Fuzzy Math., 11 (2003), 687-705. 1.1

[3] J. A. Baker, Isometries in normed spaces, Amer. Math. Monthly, 78 (1971), 655-658. 1, 3.5, 3.9

[4] S. C. Cheng, J. N. Mordeson, Fuzzy linear operators and fuzzy normed linear spaces, Bull. Calcutta Math. Soc., 86 (1994), 429-436. 1.1

[5] C. Felbin, Finite-dimensional fuzzy normed linear space, Fuzzy Sets and Systems, 48 (1992), 239-248. 1.1

[6] J. J. Font, J. Galindo, S. Macario, M. Sanchis, A generalized Mazur-Ulam theorem for fuzzy normed spaces, Abstr. Appl. Anal., 2014 (2014), 4 pages. 1, 3.5

[7] V. Gregori, S. Romaguera, Some properties of fuzzy metric spaces, Fuzzy Sets and Systems, 115 (2000), 485-489. 1.1

[8] O. Kaleva, S. Seikkala, On fuzzy metric spaces, Fuzzy Sets and Systems, 12 (1984), 215-229. 1.1

[9] A. K. Katsaras, Fuzzy topological vector spaces, II, Fuzzy Sets and Systems, 12 (1984), 143-154. 1.1

[10] I. Kramosil, J. Michálek, Fuzzy metrics and statistical metric spaces, Kybernetika (Prague), 11 (1975), 336-344. 1.1, 1.1

[11] S. Mazur, S. Ulam, Sur les transformations isométriques d'espaces vectoriels normés, CR Acad. Sci. Paris, 194 (1932), 946-948. 1

[12] H. Rui, On the maps preserving the equality of distance, J. Math. Anal. Appl., 343 (2008), 1161-1165. 1, 3.5

[13] I. J. Schoenberg, Metric spaces and completely monotone functions, Ann. of Math., 39 (1938), 811-841. 3.5

[14] B. Schweizer, A. Sklar, Statistical metric spaces, Pacific J. Math., 10 (1960), 313-334. 1.1

[15] A. Vogt, Maps which preserve equality of distance, Studia Math., 45 (1973), 43-48. 1, 3.5

[16] J. von Neumann, I. J. Schoenberg, Fourier integrals and metric geometry, Trans. Amer. Math. Soc., 50 (1941), $226-251$. $1,3.5$ 